\title{
STUDY OF GENTIC PARAMETERS USING HALF DIALLEL CROSS IN MAIZE (ZEA MAYS L.)
}

\author{
Mohammed S. Altaweel \\ Qusay A. Yousef
}

Field Crop Dept., College of Agriculture and Forestry, University of Mosul, Iraq

Emaildraltwel@uomosul.edu.iq

\begin{abstract}
Five inbred lines of maize (Zea mays L.) were used in this study with ten hybrids which planted through spring and autumn seasons (2018) at the research station of the Field Crops Department/College of Agriculture and Forestry/University of Mosul, using Randomized Complete Block Design with three replications. The characters studied were date to tasseling, date to silking, plant height, number of leaves per plant, leaf area $\mathrm{cm}^{2}$, leave area index, number of ears per plant, ear length, number of rows per ear, number grains per row, 300 grain weight, grain yield per plant and oil percent \%.The results showed that phenotypic, genetic and environmental variances were significant for all the characters for both seasons. The average degree of dominance was greater than one for number of leaves per plant, leaf area index, number of ears per plant, ear length, number of rows per ear, the number grains per row and oil percent. The broad sense heritability was high for plant height, leaf area, leaf area index, number of rows per ear, 300 grain weight, grain yield per plant, and oil percent for both seasons, while it was medium for number of leaves per plant, number of ears per plant, ear length, number grain per row, and low for date to tasseling, date to silking, at autumn season. The expected genetic advance values for all characters and for both seasons was high.
\end{abstract}

Key words: maize, phenotypic and genetic Variance, Average degree of dominance.

Received: 16 / 9 / 2020, Accepted: 10 / 10 / 2020

\section{INTRODUCTION}

Maize belongs to the Poaceae family and the Maydeae tribe and it occupies the second place in terms of importance after wheat and first in terms of production In Iraq. The highest cultivated area using this crop reached 55 thousand dunums and produced 63.3 thousand tons of grain with an average yield of (1133.8) $\mathrm{kg} / \mathrm{dunum}$ (Central Statistical Organization 2018). The continued genetic progress in breeding programs maize depends on the formation of new, unfamiliar consortia in order to obtain genetic parameters which have new traits and desirable for plant breeders. Bandar (2016) during her study of the maize obtained that there are significant differences among Genotypes and genetic variance values were greater than the environmental variation in the characters of date to silking, date to tasseling, plant height, leaf area, number of rows, number of grains per row, 300 grains weight, grain yield per plant (gm). Hady et al., (2004); Mualla et al., (2011) and Hady et al., (2017) showed through their experiment on the maize crop, that the genetic variances were higher than the environmental variances for plant height, number of leaves, leaf area, ear length, number of rows per ear, number of grains per row and number of ear. Therefore, the heritability values in the broad sense were all high. 
On the other hand, Saadallah et al., (2017); Li (2004) and Al Bank (2009) explained that the average degree of dominance was greater than one for most of the studied traits. Bandar (2016) noted that the values of broad sense heritability were high for most studied traits. Ali et al., (2006); Najeeb et al., (2009); Al Bank (2009) and Anis (2010) reported that the expected genetic improvement was high for some traits and medium for others, while it was low for some other traits. Ali et al., (2019), during their study of the maize found that the values of heritability in a broad sense were high for the characters of date to silking, date to tasseling, plant height, and medium for two characters of the number of grains per row and grain yield per plant. This study aims to estimate the genetic parameters which include Genetic, phenotypic, environment variances, average degree of dominance, broad and narrow sense heritability, and the expected genetic improvement.

\section{MATRIALS AND METHODS}

Five pure lines of maize (Zea mays L) with their ten half diallel crosses were used in this study whose origin is Dr. Muhammad Al-Falahi (Faculty of Agriculture and Forestry - University of Dohuk), the name of pure lines and origin are shown in Table (1).

Table (1): Name of pure lines used in this study and their origin.

\begin{tabular}{|c|c|c|}
\hline No. & Genotypes & Origin \\
\hline 1 & DKcc6418 & American \\
\hline 2 & Veges From pohene & France \\
\hline 3 & HS & Aebaa center \\
\hline 4 & Un44052 & Uonan \\
\hline 5 & IK58 & Aebaa center \\
\hline
\end{tabular}

The seeds of the genotypes were sown on 15/3/2018 for the spring season and on $15 / 7 / 2018$ for the autumn season at the research station of Field Crops Department/College of Agriculture and Forestry/University of Mosul using randomized complete block design (R.C.B.D) with three replications, where the seeds of each genotype sown in two rows each of 2 meter length, and the distance between them is $(0.75) \mathrm{m}$ and between plants $(0.25 \mathrm{~m})$. Two seeds were placed in each groove and then thinned to one plant. Soil and crop service operations were carried out as recommendation and the weeds were controlled using Paraquat pesticide concentration $(20 \%)$ with a quantity of $20 \mathrm{ml}$ per 20 liters of water two times. The first one was after two weeks of planting and the second after two weeks of the first control. Ten plants were selected randomly from each experimental unit take measurements. The data were recorded for the following traits: date to tasseling, date to silking, plant height $(\mathrm{cm})$, number of leaves per plant, leaf area $\mathrm{cm}^{2}$, leave area index, number of ears per plant, ear length $(\mathrm{cm})$, number of rows per ear, number grains per row, 300 grain weight (gm), grain yield per plant (gm/plant) and oil percent $\%$.The statistical analysis of the data was carried out according to the method of the used (Al-Rawi and Khalaf Allah , 2000) and with the help of the SAS program. The genetic and environmental variances, the genetic coefficient of 
variability, the phenotypic coefficient of variability, and the broad sense heritability were estimated the way which is explained by Singh and Chaudhary (2000).

$$
\begin{aligned}
\sigma_{g}^{2} & =(M . S . t-M . s . e) / r & \sigma^{2}{ }_{e}=\text { M.S.e } & \sigma_{p}^{2}=\sigma_{g}^{2}+\sigma_{e}^{2} \\
\mathrm{H}_{(\mathrm{N} . \mathrm{S})} & =(\mathrm{VA} / \mathrm{VP}) \times 100 & &
\end{aligned}
$$

The ranges of narrow sense heritability value as reported by Ali (1999) were: less than $40 \%$ low, 40-60\% moderate, and more than $60 \%$ high. The expected genetic improvement was estimated when $5 \%$ of the plants were selected, according to Johansson et al., (1955).

G.A.\% $=\frac{G . A}{\bar{Y}} \times 100 \quad$ G.A $=i \sqrt{\sigma_{P}^{2}} H^{2}$

Where $\mathrm{i}=$ selection intensity $=2.06$

The ranges suggested by Agarwal and Ahmad (1982) were used for predicted genetic improvement as a percentage of the mean: less than $10 \%$ low, between 10$30 \%$ moderate and more than $30 \%$ is high. The phenotypic variance was estimated from the sum of the genetic and environmental variances assuming the absence of genetic-environmental interaction (Falconer, 1964) and the standard error of genetic and environmental variance was estimated according to what Kempthorne (1957) stated according to the following formula:

$S E\left(\sigma^{2} G\right)=\sqrt{\frac{1}{r^{2}}\left[\frac{2(m s g)^{2}}{k+2}+\frac{2(m s e)^{2}}{k+2}\right]} \quad S E\left(\sigma^{2} E\right)=\sqrt{\frac{2(m s e)^{2}}{k+2}} \quad S E\left(\sigma^{2} p\right)=\sqrt{\frac{2\left(\sigma^{2} p\right)^{2}}{N}}$

Noting that: $\mathrm{k}=$ degrees of freedom for each source (genotype or experimental error).

$\mathrm{r}=$ number of repeats, whereas the standard error of phenotypic anisotropy was estimated according to Mather and Jinks (1981). Where, $\mathrm{N}=$ the sum of the degrees of freedom for genotypes and experimental error.

\section{RESULTS AND DISCUSSION}

Table (2) shows the results of the analysis of variance for studied traits at spring season. It is noted that the mean squares of genotypes was significant at a probability level of $1 \%$ for all the studied traits. This is considered an important to continuing the genetic analysis and the estimation of the components of variance and genetic parameters in order to identify the appropriate breeding method to improve the crop.

Table (3) shows the analysis of variance results at autumn season: It is noticed that average of squares the genotypes was significant at $1 \%$ probability level for the characters of plant height, number of leaves per plant, leaf area $(\mathrm{cm})$, leaf area index, number of ears per plant, length of ear, number of rows per ear, number grains per row, grains 300 weight, single plant yield, ratio oil (\%), and significant at $5 \%$ probability level for the traits of date to silking, date to tasseling. The significant difference among the genotypes provides the possibility to select the superior genotype. 
Mesopotamia J. of Agric.

Vol. (48) No. (4) 2020
ISSN: 2224 - 9796 (Online)

ISSN: 1815 - 316 X (Print)
مجلــــة زر اعــة الــر افديـن

المجلد (48) العدد (4) 2020

Table (2): Analysis of variance results for studied traits of maize at spring season.

\begin{tabular}{|c|c|c|c|c|c|c|c|c|}
\hline S.O.V & d.f & $\begin{array}{l}\text { Date to } \\
\text { silking }\end{array}$ & $\begin{array}{l}\text { Date } \\
\text { to } \\
\text { tasseli } \\
\text { ng }\end{array}$ & $\begin{array}{l}\text { Plant } \\
\text { high } \\
(\mathrm{cm})\end{array}$ & $\begin{array}{l}\text { Numbe } \\
\text { r of } \\
\text { leaves } \\
\text { per } \\
\text { plant }\end{array}$ & $\begin{array}{l}\text { Leaf area } \\
\quad(\mathrm{cm} 2)\end{array}$ & $\begin{array}{l}\text { Leaf } \\
\text { area } \\
\text { index }\end{array}$ & $\begin{array}{c}\text { Numbe } \\
\text { r of } \\
\text { ears } \\
\text { per } \\
\text { plant }\end{array}$ \\
\hline Block & 2 & 2.467 & 2.467 & 1.140 & 0.751 & $\begin{array}{c}584112.52 \\
6\end{array}$ & 0.030 & 0.057 \\
\hline $\begin{array}{c}\text { Treatme } \\
\text { nt }\end{array}$ & 14 & $\begin{array}{c}* * \\
49.619\end{array}$ & $\begin{array}{c}* * * \\
61.67 \\
6\end{array}$ & $\begin{array}{c}* * \\
297.20 \\
7\end{array}$ & $\begin{array}{c}* * \\
0.371\end{array}$ & $\begin{array}{c}* * * \\
466784.94 \\
3 \\
\end{array}$ & $\begin{array}{c}* * \\
0.186\end{array}$ & $\begin{array}{c}* * \\
0.123\end{array}$ \\
\hline Error & 28 & 6.362 & 2.005 & 4.052 & 0.074 & 77362.773 & 0.024 & 0.025 \\
\hline
\end{tabular}

\section{Follows Table (2)}

\begin{tabular}{|c|c|c|c|c|c|c|c|}
\hline S.O.V & d.f & $\begin{array}{c}\text { ear } \\
\text { length }(\mathrm{c} \\
\mathrm{m})\end{array}$ & $\begin{array}{l}\text { number } \\
\text { of rows } \\
\text { per ear }\end{array}$ & $\begin{array}{l}\text { number } \\
\text { grains per } \\
\text { row }\end{array}$ & $\begin{array}{c}300 \\
\text { grains } \\
\text { weight } \\
(\mathrm{gm})\end{array}$ & $\begin{array}{l}\text { grain } \\
\text { yield per } \\
\text { plant }\end{array}$ & $\begin{array}{c}\text { Oil percent } \\
\%\end{array}$ \\
\hline Block & 2 & 0.106 & 0.434 & 0.828 & 14.099 & 8.928 & 2.917 \\
\hline treatment & 14 & $\begin{array}{c}* * \\
29.154\end{array}$ & $\begin{array}{c}* * \\
1.474\end{array}$ & $\begin{array}{c}* * \\
36.521\end{array}$ & $\begin{array}{c}* * \\
165.927\end{array}$ & $\begin{array}{c}* * \\
780.670\end{array}$ & $\begin{array}{c}* * \\
0.525\end{array}$ \\
\hline Error & 28 & 0.230 & 0.109 & 0.907 & 7.942 & 2.798 & 0.020 \\
\hline
\end{tabular}

** Significant of level probability $1 \%$

Table (3): Analysis of variance using full randomized sector design.

\begin{tabular}{|c|c|c|c|c|c|c|c|c|}
\hline S.O.V & d.f & $\begin{array}{l}\text { Date to } \\
\text { silking }\end{array}$ & $\begin{array}{l}\text { Date to } \\
\text { tasselin } \\
\quad \mathrm{g}\end{array}$ & $\begin{array}{l}\text { Plant } \\
\text { high } \\
(\mathrm{cm})\end{array}$ & $\begin{array}{l}\text { numb } \\
\text { er of } \\
\text { leave } \\
\text { s per } \\
\text { plant }\end{array}$ & $\begin{array}{l}\text { Leaf area } \\
\quad\left(\mathrm{cm}^{2}\right)\end{array}$ & $\begin{array}{l}\text { Leaf } \\
\text { area } \\
\text { index }\end{array}$ & $\begin{array}{c}\text { numbe } \\
\text { r of } \\
\text { ears } \\
\text { per } \\
\text { plant }\end{array}$ \\
\hline Block & 2 & $\begin{array}{c}175.40 \\
0\end{array}$ & $\begin{array}{c}153.15 \\
6\end{array}$ & 234.380 & 1.072 & 37294.264 & 0.005 & 0.123 \\
\hline $\begin{array}{c}\text { treatmen } \\
\mathrm{t}\end{array}$ & 14 & $\begin{array}{c}* \\
48.943\end{array}$ & $\begin{array}{c}* * \\
50.603\end{array}$ & $\begin{array}{c}* * \\
321.263\end{array}$ & $\begin{array}{c}* * \\
0.377\end{array}$ & $\begin{array}{c}* * * \\
459862.46 \\
4\end{array}$ & $\begin{array}{c}* * \\
0.121\end{array}$ & $\begin{array}{c}* * \\
0.046\end{array}$ \\
\hline Error & 28 & 18.300 & 18.290 & 45.751 & 0.092 & 44184.595 & 0.017 & 0.012 \\
\hline
\end{tabular}


Mesopotamia J. of Agric.

Vol. (48) No. (4) 2020
ISSN: 2224 - 9796 (Online)

ISSN: 1815 - $316 \mathrm{X}$ (Print)

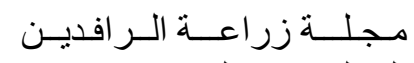

المجلد (48) العدد (4) 2020

Follow table 3

\begin{tabular}{|c|c|c|c|c|c|c|c|}
\hline S.O.V & d.f & $\begin{array}{c}\text { ear } \\
\text { length }( \\
\mathrm{cm})\end{array}$ & $\begin{array}{l}\text { number } \\
\text { of rows } \\
\text { per ear }\end{array}$ & $\begin{array}{l}\text { number } \\
\text { grains } \\
\text { per row }\end{array}$ & $\begin{array}{c}300 \text { grains } \\
\text { weight } \\
(\mathrm{gm})\end{array}$ & $\begin{array}{c}\text { grain } \\
\text { yield per } \\
\text { plant }\end{array}$ & $\begin{array}{c}\text { oil } \\
\text { percent } \\
\% .\end{array}$ \\
\hline Block & 2 & 19.708 & 268.447 & 12.779 & 6.362 & 94.316 & 0.694 \\
\hline treatment & 14 & $\begin{array}{c}* * \\
8.960\end{array}$ & $\begin{array}{c}* * \\
274.434\end{array}$ & $\begin{array}{c}* * \\
54.931\end{array}$ & $\begin{array}{c}* * \\
1087.684\end{array}$ & $\begin{array}{c}* * \\
1523.184\end{array}$ & $\begin{array}{c}* * \\
0.888\end{array}$ \\
\hline Error & 28 & 2.323 & 79.922 & 9.212 & 79.823 & 168.179 & 0.061 \\
\hline
\end{tabular}

** Significant of level probability $1 \%$

Table (4): Components of variance and genetics parameters for studies traits at spring season.

\begin{tabular}{|c|c|c|c|c|c|c|c|}
\hline $\begin{array}{c}\text { Genetic } \\
\text { parameters }\end{array}$ & $\begin{array}{l}\text { Date to } \\
\text { silking }\end{array}$ & $\begin{array}{l}\text { Date to } \\
\text { tasseling }\end{array}$ & $\begin{array}{l}\text { Plant } \\
\text { high } \\
(\mathrm{cm})\end{array}$ & $\begin{array}{c}\text { number } \\
\text { of } \\
\text { leaves } \\
\text { per plant }\end{array}$ & $\begin{array}{l}\text { Leaf area } \\
\left(\mathrm{cm}^{2}\right)\end{array}$ & $\begin{array}{l}\text { Leaf } \\
\text { area } \\
\text { index }\end{array}$ & $\begin{array}{l}\text { number } \\
\text { of ears } \\
\text { per plant }\end{array}$ \\
\hline $\begin{array}{l}\text { Additive } \\
\text { variance }\end{array}$ & $\begin{array}{l}4.831 \\
\quad \pm \\
6.364\end{array}$ & $\begin{array}{l}3.256 \\
\pm \\
2.008\end{array}$ & $\begin{array}{l}94.479 \\
\pm \\
5.353\end{array}$ & $\begin{array}{c}0.135 \\
\pm \\
0.074\end{array}$ & $\begin{array}{c}225369.96 \\
5 \\
\pm \\
77811.771\end{array}$ & $\begin{array}{c}0.074 \\
\pm \\
0.024\end{array}$ & $\begin{array}{c}0.024 \\
\pm \\
0.024\end{array}$ \\
\hline $\begin{array}{c}\text { Dominance } \\
\text { variance }\end{array}$ & $\begin{array}{l}68.501 \\
\quad \pm \\
23.703\end{array}$ & $\begin{array}{c}85.695 \\
\pm \\
28.635\end{array}$ & $\begin{array}{c}397.19 \\
4 \\
\pm \\
132.46 \\
0\end{array}$ & $\begin{array}{l}0.492 \\
\quad \pm \\
0.180\end{array}$ & $\begin{array}{c}608424.92 \\
7 \\
\pm \\
217062.68 \\
4\end{array}$ & $\begin{array}{c}0.246 \\
\pm \\
0.085\end{array}$ & $\begin{array}{c}0.168 \\
\pm \\
0.061\end{array}$ \\
\hline $\begin{array}{c}\text { Phenotypic } \\
\text { variance }\end{array}$ & $\begin{array}{c}20.781 \\
\pm \\
1.642\end{array}$ & $\begin{array}{c}21.895 \\
\pm \\
4.7779\end{array}$ & $\begin{array}{c}101.77 \\
0 \\
\pm \\
22.208\end{array}$ & $\begin{array}{c}0.173 \\
\pm \\
0.037\end{array}$ & $\begin{array}{c}207170.16 \\
3 \\
\pm \\
45208.235 \\
\end{array}$ & $\begin{array}{c}0.078 \\
\pm \\
0.017\end{array}$ & $\begin{array}{c}0.058 \\
\pm \\
0.012\end{array}$ \\
\hline $\begin{array}{c}\text { Environme } \\
\text { ntal } \\
\text { variance }\end{array}$ & 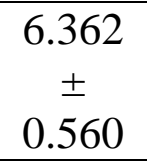 & $\begin{array}{c}2.005 \\
\pm \\
0.998 \\
\end{array}$ & $\begin{array}{c}4.052 \\
\pm \\
0.702 \\
\end{array}$ & $\begin{array}{c}0.074 \\
\pm \\
5.200 \\
\end{array}$ & $\begin{array}{c}77362.773 \\
\pm \\
0.005 \\
\end{array}$ & $\begin{array}{c}0.024 \\
\pm \\
9.138 \\
\end{array}$ & $\begin{array}{c}0.025 \\
\pm \\
8.997 \\
\end{array}$ \\
\hline $\begin{array}{c}\text { Average } \\
\text { degree } \\
\text { dominance }\end{array}$ & 2.423 & 4.021 & 0.298 & 7.376 & 0.005 & 9.488 & 24.204 \\
\hline $\begin{array}{c}\text { Broad } \\
\text { seance } \\
\text { heritability }\end{array}$ & 69.386 & 90.844 & 96.019 & 57.243 & 62.657 & 69.309 & 57.047 \\
\hline $\begin{array}{c}\text { Narrow } \\
\text { seance } \\
\text { heritability }\end{array}$ & 0.060 & 0.035 & 0.190 & 0.019 & 0.247 & 0.215 & 0.110 \\
\hline
\end{tabular}


Mesopotamia J. of Agric.

Vol. (48) No. (4) 2020
ISSN: 2224 - 9796 (Online)

ISSN: 1815 - $316 \mathrm{X}$ (Print)

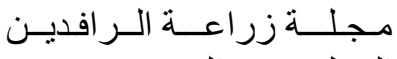

المجلد (48) العدد (4) 2020

\begin{tabular}{|c|c|c|c|c|c|c|c|}
\hline $\begin{array}{c}\text { Expected } \\
\text { genetic } \\
\text { improveme } \\
\text { nt }\end{array}$ & 6.515 & 8.756 & 19.954 & 0.490 & 587.493 & 0.398 & 0.281 \\
\hline $\begin{array}{c}\text { Expected } \\
\text { genetic } \\
\text { improveme } \\
\text { nt } \%\end{array}$ & 9.220 & 10.909 & 12.539 & 4.546 & 16.938 & 21.186 & 23.524 \\
\hline
\end{tabular}

Follows Table (4):

\begin{tabular}{|c|c|c|c|c|c|c|}
\hline $\begin{array}{c}\text { Genetic } \\
\text { parameters }\end{array}$ & $\begin{array}{c}\text { ear } \\
\text { length }(\mathrm{cm})\end{array}$ & $\begin{array}{l}\text { number } \\
\text { of rows } \\
\text { per ear }\end{array}$ & $\begin{array}{l}\text { number } \\
\text { grains per } \\
\text { row }\end{array}$ & $\begin{array}{c}300 \\
\text { grains } \\
\text { weight } \\
(\mathrm{gm})\end{array}$ & $\begin{array}{l}\text { grain } \\
\text { yield per } \\
\text { plant }\end{array}$ & $\begin{array}{c}\text { Oil } \\
\text { percent } \\
\%\end{array}$ \\
\hline $\begin{array}{l}\text { Additive } \\
\text { variance }\end{array}$ & $\begin{array}{c}4.294 \\
\pm \\
0.280\end{array}$ & $\begin{array}{c}0.414 \\
\pm \\
0.110\end{array}$ & $\begin{array}{c}3.766 \\
\pm \\
0.917\end{array}$ & $\begin{array}{c}36.146 \\
\pm \\
8.053\end{array}$ & $\begin{array}{c}131.974 \\
\pm \\
5.632\end{array}$ & $\begin{array}{c}0.175 \\
\pm \\
0.020\end{array}$ \\
\hline $\begin{array}{c}\text { Dominance } \\
\text { variance }\end{array}$ & $\begin{array}{c}39.957 \\
\pm \\
13.321\end{array}$ & $\begin{array}{c}1.981 \\
\pm \\
0.669\end{array}$ & $\begin{array}{c}50.376 \\
\pm \\
168.816\end{array}$ & $\begin{array}{c}212.468 \\
\pm \\
71.266\end{array}$ & $\begin{array}{c}1066.543 \\
\pm \\
355.525\end{array}$ & $\begin{array}{c}0.701 \\
\pm \\
0.234\end{array}$ \\
\hline $\begin{array}{c}\text { Phenotypic } \\
\text { variance }\end{array}$ & $\begin{array}{c}0.564 \\
\pm \\
0.123\end{array}$ & $\begin{array}{c}12.778 \\
\pm \\
2.788\end{array}$ & $\begin{array}{c}9.872 \\
\pm \\
2.154\end{array}$ & $\begin{array}{c}57.603 \\
\pm \\
1.506\end{array}$ & $\begin{array}{c}262.089 \\
\pm \\
57.192\end{array}$ & $\begin{array}{c}0.188 \\
\pm \\
0.0411\end{array}$ \\
\hline $\begin{array}{c}\text { Environme } \\
\text { ntal } \\
\text { variance } \\
\end{array}$ & $\begin{array}{c}0.230 \\
\pm \\
2.946\end{array}$ & $\begin{array}{c}0.109 \\
\pm \\
4.274\end{array}$ & $\begin{array}{c}0.907 \\
\pm \\
1.484\end{array}$ & $\begin{array}{c}7.942 \\
\pm \\
0.501\end{array}$ & $\begin{array}{c}2.798 \\
\pm \\
0.845\end{array}$ & $\begin{array}{c}0.020 \\
\pm \\
10.124 \\
\end{array}$ \\
\hline $\begin{array}{c}\text { Average } \\
\text { degree } \\
\text { dominance }\end{array}$ & 2.082 & 4.807 & 2.665 & 0.570 & 0.350 & 6.759 \\
\hline $\begin{array}{c}\text { Broad } \\
\text { seance } \\
\text { heritability }\end{array}$ & 80.621 & 92.901 & 97.666 & 86.213 & 98.932 & 89.630 \\
\hline $\begin{array}{c}\text { Narrow } \\
\text { seance } \\
\text { heritability }\end{array}$ & 0.165 & 0.068 & 0.096 & 0.140 & 0.109 & 0.195 \\
\hline $\begin{array}{c}\text { Expected } \\
\text { genetic } \\
\text { improveme } \\
\text { nt }\end{array}$ & 1.247 & 6.841 & 6.321 & 13.479 & 32.993 & 0.800 \\
\hline $\begin{array}{c}\text { Expected } \\
\text { genetic } \\
\text { improveme } \\
\text { nt \% }\end{array}$ & 9.323 & 34.460 & 35.995 & 16.090 & 44.457 & 27.351 \\
\hline
\end{tabular}


Table (4) shows the components of variance and the genetic parameters for studied traits at spring season, it is noticed that the additive and dominance genetic variances were significant from zero for all traits. This is consistent with Hady and Waheeb (2004), Mualla et al., (2011) and Hadi et al., (2017). It is also noticed that the average degree of dominance was greater than one for date to silking, date to tasseling, number of leaves per plant, leaf area index, number of ears per plant, ear length, number of rows per ear, number of grains per row and ratio oil\%. So, we conclude the existence of over dominance, while it was less than one for plant height, leaf area, grains 300 weight, and single yield plant, so we conclude the existence of partial dominance, and this is consistent with what was found by $\mathrm{Li}$ (2004) and Al Bank (2009) and Saadallah et al., (2017). It was noticed that broad sense heritability values were high for all the studied traits, except that it was moderate for number of leaves per plant and number ears per plant, and this is consistent with Al Hadi et al., (2013) Bandar (2016) and Saad Allah et al., (2017). It was found in the table (4) also, that narrow sense heritability values was low for other traits, and this is consistent with the findings of the researchers Chohan et al., (2012), Al-Hadi et al., (2013), Saad Allah et al., (2017) and Kanoush (2018). It was shown that the expected genetic improvement was high for leaf area, single yield plant $(\mathrm{gm} / \mathrm{plant})$, medium for plant height $(\mathrm{cm})$, and grains 300 weight $(\mathrm{gm})$, and was low for date to silking and date to tasseling, number of leaves per plant, of leaf area index, and number ears per plant, ear length $(\mathrm{cm})$, number of rows per ear, number of grains per row, ratio oil (\%). This is consistent with Ali et al. (2006), Shakoor et al., (2007), Najeeb et al. (2009), Al Bank (2009), and Anis. (2010).

Table (5) shows the components of variation and the genetic parameters of the studied traits at autumn season. It was found that additive and dominance genetic variances were significant from aero for all traits, and this is consistent with what was found by Hadi and Waheeb (2004) and Mualla et al., (2011) and Hadi et al., (2017). It is also noticed that the average degree of dominance was greater than one for number of leaves per plant, Leaf area index, number of ears per plant, number grains per row and ratio of oil \% which refers to the over dominance. On the other hand, the average degree of dominance was less than one for date to tasseling, date to silking, plant height $(\mathrm{cm})$, leaf area $\left(\mathrm{cm}^{2}\right)$, length of ear $(\mathrm{cm})$, number of rows per ear, grains 300 weight (gm), and single yield plant (gm/plant). This is agreement with Bektash and Abed (2002), Al Aswadi (2002), Al-Azzawi (2002), Bektash and Al-Azzawi (2004) and Shaya (2007). It was also shown that the values of broad sense heritability were high for the traits, plant height $(\mathrm{cm})$, leaf area $\left(\mathrm{cm}^{2}\right)$, leaf area index, number of rows per ear, grains 300 weight $(\mathrm{gm})$, single yield plant (gm/plant) and ratio oil $(\%)$, and it was moderate for the number of leaves, the number of ear per plant, the length of ear $(\mathrm{cm})$ and the number of grains per row. While, it was low for date to silking and date to tasseling, this is agreement with Al-Hadi et al., (2013), Bandar (2016), Saad Allah et al., (2017) and Kanoush (2018). It was found in the same table that the values of the narrow sense heritability were low for all the studied traits, and this is consistent with the findings of scholars Chohan et al., (2012), Al-Hadi et al., (2013), Saad Allah et al., (2017) and Kanoush (2018). It shows that the expected genetic improvement is high for the characteristic of leaf area, medium for the characteristic of plant height, 300 grains 
Mesopotamia J. of Agric. Vol. (48) No. (4) 2020
ISSN: 2224 - 9796 (Online)

ISSN: 1815 - 316 X (Print)

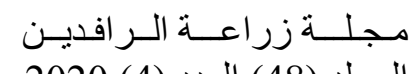

weight, single yield plant (gm/plant), but it was low for date to silking, date to tasseling, number of leaves per plant, leaf area index and number ears per plant, number of rows in ear, ear length $(\mathrm{cm})$, number of grains per row, and ratio oil $(\%)$. This is agreement with Ali et al., (2006), Shakoor et al., (2007), Najeeb et al., (2009),Al Bank (2009) and Anis (2010).

The significant differences between inbred lines provide the opportunity for a selection between these strains, as inbred lines (DKcc6418) was the best for (number of rows per ear, number grains per row, 300 grains weight, and grain yield per plant for the spring season, while the inbred lines (HS) was the best for most of the traits, number of rows per ear grain yield per plant for the autumn season, and thus we recommend that you take advantage of them by following introduction steps to obtain a new variety or hybridize them with local varieties to transfer the yield genes and other traits

Table (5): Components of variance and genetics parameters for studies traits at autumn season.

\begin{tabular}{|c|c|c|c|c|c|c|c|}
\hline $\begin{array}{c}\text { Genetic } \\
\text { parameters }\end{array}$ & $\begin{array}{l}\text { Date } \\
\text { to } \\
\text { silking }\end{array}$ & $\begin{array}{l}\text { Date to } \\
\text { tasselin } \\
\text { g }\end{array}$ & $\begin{array}{l}\text { Plant high } \\
\text { (cm) }\end{array}$ & $\begin{array}{l}\text { number } \\
\text { of leaves } \\
\text { per plant }\end{array}$ & $\begin{array}{l}\text { Leaf area } \\
\qquad\left(\mathrm{cm}^{2}\right)\end{array}$ & $\begin{array}{l}\text { Leaf } \\
\text { area } \\
\text { index }\end{array}$ & $\begin{array}{c}\text { number } \\
\text { of ears } \\
\text { per } \\
\text { plant }\end{array}$ \\
\hline $\begin{array}{l}\text { Additive } \\
\text { variance }\end{array}$ & $\begin{array}{l}15.109 \\
\quad \pm \\
18.309\end{array}$ & $\begin{array}{l}16.360 \\
\quad \pm \\
18.300\end{array}$ & $\begin{array}{c}73.355 \\
\pm \\
45.355\end{array}$ & $\begin{array}{c}0.125 \\
\pm \\
0.092\end{array}$ & $\begin{array}{c}134934.7 \\
43 \\
\pm \\
44466.32 \\
7\end{array}$ & $\begin{array}{c}0.033 \\
\pm \\
0.017\end{array}$ & $\begin{array}{c}0.023 \\
\pm \\
0.011\end{array}$ \\
\hline $\begin{array}{c}\text { Dominance } \\
\text { variance }\end{array}$ & $\begin{array}{l}65.498 \\
\quad \pm \\
28.488\end{array}$ & $\begin{array}{l}67.572 \\
\quad \pm \\
29.014\end{array}$ & $\begin{array}{c}435.097 \\
\pm \\
152.077\end{array}$ & $\begin{array}{c}0.503 \\
\pm \\
0.191\end{array}$ & $\begin{array}{c}616820.5 \\
00 \\
\pm \\
210300.8 \\
52\end{array}$ & $\begin{array}{c}0.163 \\
\pm \\
0.057\end{array}$ & $\begin{array}{c}0.059 \\
\pm \\
0.023\end{array}$ \\
\hline $\begin{array}{c}\text { Phenotypic } \\
\text { variance }\end{array}$ & $\begin{array}{l}28.514 \\
\pm \\
4.8082\end{array}$ & $\begin{array}{c}29.061 \\
\pm \\
6.341\end{array}$ & $\begin{array}{c}137.859 \\
\pm \\
30.024\end{array}$ & $\begin{array}{c}0.187 \\
\pm \\
0.040\end{array}$ & $\begin{array}{c}182743.8 \\
84 \\
\pm \\
39877.98 \\
4\end{array}$ & $\begin{array}{c}0.052 \\
\pm \\
0.011\end{array}$ & $\begin{array}{c}0.023 \\
\pm \\
0.005\end{array}$ \\
\hline $\begin{array}{l}\text { Environmen } \\
\text { tal variance }\end{array}$ & $\begin{array}{c}18.300 \\
\pm \\
0.330\end{array}$ & $\begin{array}{l}18.290 \\
\pm \\
0.331\end{array}$ & $\begin{array}{c}45.751 \\
\pm \\
0.209\end{array}$ & $\begin{array}{c}0.092 \\
\pm \\
4.652\end{array}$ & $\begin{array}{c}44184.59 \\
5 \\
\pm \\
0.006\end{array}$ & $\begin{array}{c}0.017 \\
\pm \\
10.710\end{array}$ & $\begin{array}{c}0.012 \\
\pm \\
12.989\end{array}$ \\
\hline $\begin{array}{c}\text { Average } \\
\text { degree } \\
\text { dominance }\end{array}$ & 0.758 & 0.711 & 0.402 & 8.048 & 0.008 & 17.456 & 14.731 \\
\hline $\begin{array}{c}\text { Broad } \\
\text { seance } \\
\text { heritability }\end{array}$ & 35.822 & 37.064 & 66.748 & 50.681 & 75.822 & 66.510 & 48.810 \\
\hline
\end{tabular}


Mesopotamia J. of Agric.

Vol. (48) No. (4) 2020
ISSN: 2224 - 9796 (Online)

ISSN: 1815 - 316 X (Print)
مجلــــة زر اعــة الــر افديـن

المجلد (48) العدد (4) 2020

\begin{tabular}{|c|c|c|c|c|c|c|c|}
\hline $\begin{array}{c}\text { Narrow } \\
\text { seance } \\
\text { heritability }\end{array}$ & 0.814 & 0.821 & 0.132 & 0.173 & 0.169 & 0.153 & 0.247 \\
\hline $\begin{array}{c}\text { Expected } \\
\text { genetic } \\
\text { improveme } \\
\text { nt }\end{array}$ & 3.940 & 4.115 & 16.128 & 0.451 & 667.700 & 0.312 & 0.153 \\
\hline $\begin{array}{c}\text { Expected } \\
\text { genetic } \\
\text { improveme } \\
\text { nt } \%\end{array}$ & 7.324 & 6.476 & 9.361 & 4.143 & 16.327 & 14.258 & 14.781 \\
\hline
\end{tabular}

Follows Table (5)

\begin{tabular}{|c|c|c|c|c|c|c|}
\hline $\begin{array}{c}\text { Genetic } \\
\text { parameters }\end{array}$ & $\begin{array}{l}\text { ear length } \\
\quad(\mathrm{cm})\end{array}$ & $\begin{array}{l}\text { number of } \\
\text { rows per } \\
\text { ear }\end{array}$ & $\begin{array}{c}\text { number } \\
\text { grains per } \\
\text { row }\end{array}$ & $\begin{array}{l}300 \\
\text { grains } \\
\text { weight } \\
\text { (gm) }\end{array}$ & $\begin{array}{l}\text { grain } \\
\text { yield per } \\
\text { plant }\end{array}$ & $\begin{array}{c}\text { Oil percent } \\
\% .\end{array}$ \\
\hline $\begin{array}{l}\text { Additive } \\
\text { variance }\end{array}$ & $\begin{array}{c}2.579 \\
\pm \\
2.325\end{array}$ & $\begin{array}{l}2.701 \\
\pm \\
1.588\end{array}$ & $\begin{array}{c}8.214 \\
\pm \\
9.217\end{array}$ & $\begin{array}{c}404.869 \\
\pm \\
81.219\end{array}$ & $\begin{array}{c}113.824 \\
\pm \\
168.232\end{array}$ & $\begin{array}{c}0.156 \\
\pm \\
0.061\end{array}$ \\
\hline $\begin{array}{c}\text { Dominance } \\
\text { variance }\end{array}$ & $\begin{array}{c}12.029 \\
\pm \\
4.634\end{array}$ & $\begin{array}{c}18.369 \\
\pm \\
6.325\end{array}$ & $\begin{array}{c}75.260 \\
\pm \\
26.724\end{array}$ & $\begin{array}{c}1441.783 \\
\pm \\
487.178\end{array}$ & $\begin{array}{c}2109.693 \\
\pm \\
723.061\end{array}$ & $\begin{array}{c}1.211 \\
\pm \\
0.408\end{array}$ \\
\hline $\begin{array}{c}\text { Phenotypic } \\
\text { variance }\end{array}$ & $\begin{array}{c}144.759 \\
\pm \\
31.589\end{array}$ & $\begin{array}{c}24.452 \\
\pm \\
5.335\end{array}$ & $\begin{array}{c}4.536 \\
\pm \\
0.989\end{array}$ & $\begin{array}{c}415.777 \\
\pm \\
90.7299\end{array}$ & $\begin{array}{c}619.848 \\
\pm \\
135.261\end{array}$ & $\begin{array}{c}0.336 \\
\pm \\
0.073\end{array}$ \\
\hline $\begin{array}{c}\text { Environmenta } \\
\text { l variance }\end{array}$ & $\begin{array}{c}2.323 \\
\pm \\
0.927\end{array}$ & $\begin{array}{c}1.586 \\
\pm \\
1.123 \\
\end{array}$ & $\begin{array}{c}9.212 \\
\pm \\
0.465 \\
\end{array}$ & $\begin{array}{c}79.823 \\
\pm \\
0.158\end{array}$ & $\begin{array}{c}168.179 \\
\pm \\
0.109\end{array}$ & $\begin{array}{c}0.061 \\
\pm \\
5.734 \\
\end{array}$ \\
\hline $\begin{array}{c}\text { Average } \\
\text { degree } \\
\text { dominance }\end{array}$ & 0.758 & 0.650 & 1.494 & 0.133 & 0.571 & 9.990 \\
\hline $\begin{array}{l}\text { Heritability of } \\
\text { broad seance }\end{array}$ & 44.790 & 62.325 & 47.778 & 80.801 & 72.868 & 81.918 \\
\hline $\begin{array}{c}\text { Broad seance } \\
\text { heritability }\end{array}$ & 0.084 & 0.088 & 0.152 & 0.210 & 0.047 & 0.109 \\
\hline $\begin{array}{c}\text { Narrow } \\
\text { seance } \\
\text { heritability } \\
\end{array}$ & 11.101 & 6.348 & 2.139 & 33.940 & 37.371 & 0.978 \\
\hline $\begin{array}{c}\text { Expected } \\
\text { genetic } \\
\text { improvement } \\
\%\end{array}$ & 83.491 & 46.158 & 14.091 & 26.951 & 45.565 & 29.388 \\
\hline
\end{tabular}


دراسة المعالم الوراثية باستخدام التهجين التبادلي النصفي في الذرة الصفراء (Zea mays L.

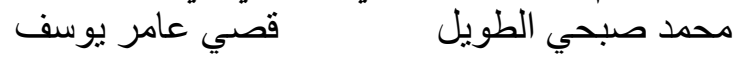

قسم المحاصيل الحقلية / كلية الزر اعة و الغابات / جامعة الموصل / العر اق

Emaildraltwel@uomosul.edu.iq

\section{الخلاصة}

استخدمت في هذه الدراسة خمس سلالات نقية من الذرة الصفراء مع هجنها النصف تبادلية العشرة حيث

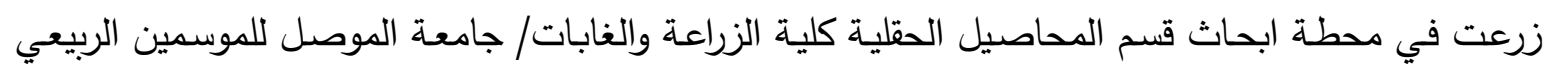

والخريفي 2018، وفق تصميم القطاعات العشوائية الكاملة بثلاث مكررات والصفات المدروسـة هي عدد الايام للتزهير الذكري (يوم) ، عدد الايام للتزهير الانثوي ، ارتفاع النبات ، عدد الاوراق بالنبات ، مساحة الورقة ،

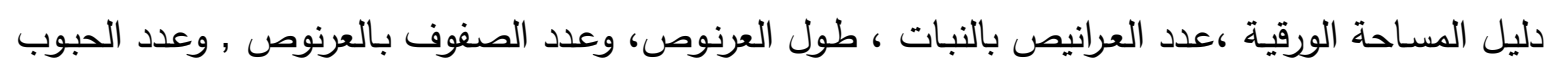

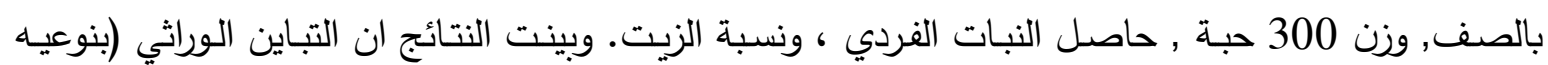
الاضافي والسيادي) والمظهري والبيئي كان معنوي لجميع الصفات ولكلا الموسمين، كان معدل درجة السيادة

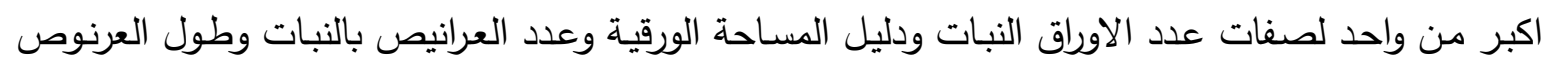
(سم) عدد الصفوف بالعرنوص وعدد الحبوب بالصف ونسبة الزيت (\%) . . كانت قيم التوريث بالمعنى الواسـع عالية لصفات ارتفاع النبات (سم) ومساحة الورقة(سم2) و دليل المساحة الورقية وعدد الصفوف بالعرنوص و وزن 300 حبة (غم) وحاصل النبات الفردي (غم) نسبة الزيت (\%) ولكلا الموسمين ، بينما كانت متوسطة عدد الأوراق بالنبات و عدد العرانيص بالنبات و طول العرنوص (سم) وعدد الحبوب بالصف في حين كانت واطئة للصفات التزهير الذكري (يوم) و التزهير الانثوي(يوم). للموسم الخريفي • وقيم التحسين الوراثي المتوقع لجميع الصفات ولكلا الموسمين كانت عالية. الكلمات المفتاحية: الذرة الصفراء، التباين الوراثي والمظهري ، معدل درجة السيادة.

$$
\text { تاريخ تسليم البحث: } 16 \text { / } 9 \text { / 2020، وقبوله : } 10 \text { / } 10 \text { / } 2020
$$

\section{REFERENCES}

Agarwal, V. and Z. Ahmad (1982). Heritability and genetic advance in triticale. Indian Journal Agriculture. Research: $19-23$.

Al Bank, L. N. M. (2009). The Nature of Gene Action by Using Half-Cross Analysis in Zea May's L. Master Thesis. Field Crops Department. College of Agriculture, University of Tikrit. Iraq.

Al-Aswadi, M. H. Y. (2002). Crossbreeding and Estimation of Genetic Landmarks, Genetic and Phenotypic Associations between Traits of Inbred Lines of maize. $\mathrm{PhD}$ thesis, Department of Field Crops. College of Agriculture. Baghdad University. Iraq.

Al-Azzawi, N. M. (2002). Genetic Analysis of First Generation Hybrids in Maize (Zea mays L). Master Thesis. Field Crops Department. College of Agriculture. Baghdad University. 
Al-Hadi, R. A ; M. Sobouh and S. Al-Ahmad (2013). Genetic analysis of some traits in isolate generations of two maize hybrids. Damascus University Journal of Agricultural Sciences 29 (2): 117-135.

Ali, A. A. Abdullah (1999). Heterosis and Gene Action in Maize (Zea mays L.), PhD thesis, College of Agriculture and Forestry, University of Mosul.

Ali, A.M. Hersi ; A.S. Ibrahim, S.H. Suliman and A.M. Suliman (2019), Genetic variability, heritability and character association of grain yield and its components among selected genotypes of maize (Zea mays L.), Gezira State, Sudan. Gezira journal of Agricultural Sciences. 17 (1).

Ali, S.; H. ur. Rahman; Raziuddin; S. S. Shah and G. Hassan. (2006). Estimates of Variability, Heritability and Genetic Advance for Fodder Traits in two Maize Populations. Pakistan Journal of Biology Sciences. 14(9): 2618-2623.

Al-Rawy, K.M. and A.K. Khalaf Allah (2000). Design and Analysis of Agricultural Experiments. Ministry of Higher Education and Scientific Research, University of Mosul.pp.1-488

Anees, A. H. A. (2010). Estimation of Genetic Landmarks in Maize (Zea mays L.) Using Single and Triple Crosses. PhD thesis. Department of Field Crops, College of Agriculture - University of Mosul, Iraq.

Baktash, F.Y. and N. M. Al-Azzawi (2004). Genetic analysis and hybrid strength of grain yield and components in maize hybrids. Iraqi Journal of Agricultural Sciences 35 (4): 67-78.

Bandar, S.J. (2016) Estimation of the genetic and phenotypic variances and correlations of a number of yellow maize traits under nitrogen abundance. Department of Field Crops, College of Agriculture - University of Baghdad. Iraqi Journal of Agricultural Sciences, 47 (4): 933-938.

Bektash, F. Y. and N. Y. Abd (2002). The ability to harmonize different genotypes of maize. Iraqi Journal of Agricultural Sciences 33 (2): 109-116.

Central Statistical Organization (2018) Annual Statistical Group, Ministry of Planning and Development Cooperation, Republic of Iraq.

Chohan, M. S.; M. S. Ahsan and M. Asghar (2012). Genetic Analysis of Water Stress Tolerance and Various Morph Physiological Traits. in Zea mays L. Using Graphical Approach. Pakistan Journal of Nutrition ISSN: 1680-5194 11(5):489-500.

Falconer, D. S. (1964). "Introduction to Quantitative Genetics". Oliver and Boyd, London, pp 129-140.

Hadi, B. H. and K. M. Wahib (2004) Heritability and Genetic Achievement in Maize. The Iraqi Agricultural Research Journal, 22: (10).96-109.

Hadi, B. H., K. M. Wahib and W. A. Hassan (2017) Estimation of genetic parameters and pathway parameters for growth traits and yield and its components for the parents and hybrids of maize. The Iraqi Agricultural Research Journal, 22: (10).142-152.

Hanson, R. G., E. Enging and E. Wing (1956). Effect of environmental factors on the fibro properties and yield of deltapine cotton. Agronomy Journal 48: 573. 
Johnson, H.w.; H.F. Robinson and R.E. Comstock (1955). Genetic and phenotypic correlation in soy bean and their implication in selection. Agronomy Journal, 47: 477- 483.

Kanoush, K. H. (2018) The ability to combine, genetic action, and heterosis in maize. Mesopotamia Journal of Agriculture 34 (4) 407-420.

Kempthorne, O. (1957). "An Introduction to Genetic Statistics". John Wiley and Sons, New York, U S A. Journal of Mammalogy, 39 (2): 313.

Li, R. (2004). A Genetic study of resistance to kernel infection by Aspergillus flavus in maize (Zea mays L.). A dissertation philosophiae doctor. The Department of agronomy and environmental management. Louisiana State University. Journal Plant Breeding, 116 (2): 146-152.

Mather, K. and J.L. Jinks , (1981). "Biometrical Genetics". 3rd Edn. New York pp. $147-162$.

Mualla, M. Y., S. A. Al-Ahmad and R. M. Hassyan (2011). Genetic variation, degree of heritability, phenotypic correlation coefficient and pathway analysis in individual hybrids in maize. Tishreen University Journal of Research and Scientific Studies - Biological Sciences Series 33 (1): 127-140.

Najeeb, S.; A. G. Rather; G. A. Parray; F. A. Sheikh and S. M Razvi. (2009). Studies on genetic variability, genotypic correlation and path coefficient analysis in maize under high altitude temperat ecology of Kashmir. Maize Genetics Cooperation Newsletter vol 83.p.1-8.

SaadAllah, H. A., M. O. H. Amin and S. A. Kakrach (2017). Complete crosscrosses for estimation of genetic parameters in maize. Journal of Agricultural Sciences, 48: 30-40.

Shakoor, M. S.; M. Akbar and A. Hussain. (2007). Correlation and Path Coefficients Studies of Some Morph physiological Traits in Maize Double Crosses. Pakistan Journal Agriculture. Sciences. 44 (2).213-216.

Shaya, H. Y. (2007). Estimation of Some Genetic Landmarks in Maize (Zea mays L.) Using Partial Cross-Analysis. Master Thesis. Department of Plant Production Techniques. Technical College. Al-Musayyib. Iraq.p.1-167

Singh, R.K. and B.D. Chaudhary (2000). "Biometrical Methods in Quantitative Genetic Analysis". Kalyani Publishers, Ludhiana, New Delhi, pp. 239-266. 